\title{
17. 14-MeV NEUTRON ACTIVATION ANALYSIS OF SELECTED LEG 5 CORE SAMPLES
}

\author{
W. E. Kuykendall, Jr., Buren W. Hoffman and R. E. Wainerdi \\ Activation Analysis Research Laboratory, Texas A\&M University \\ College Station, Texas
}

\section{SAMPLE PREPARATION}

Each core sample was first crushed and mixed to permit packaging in small polyethylene vials. Where sample size permitted, two subsamples weighing approximately 1 gram each were taken at random from the core sample, weighed and placed in vials which were then heat sealed. Standards containing known amounts of oxygen, silicon, iron, magnesium, potassium and aluminum were weighed and capsulated. Samples of the U.S.G.S. standard rock G-2, BCR-1 and GSP-1 were weighed and capsulated in a similar manner.

\section{ANALYTICAL PROCEDURES}

Each sample and standard was irradiated with $14-\mathrm{MeV}$ neutrons for two minutes, counted for two and ten minutes after cooling times of ten minutes and two hours, respectively. A Cockcroft-Walton type neutron generator provided a fast neutron flux of approximately $1 \times 10^{8} \mathrm{n} / \mathrm{an}^{2} / \mathrm{sec}$ at the sample during irradiation. A pneumatic sample transfer system was used to carry each sample and standard to the irradiation terminal and then to a 3 inch $\times 3$ inch sodium-iodide (T1) detector, which was coupled to a 400 channel pulse height analyzer. KRIS, a weighted least-squares computer program, processes the punched-tape data from the pulse height analyzer to resolve the radioactivity from the activation products of silicon, aluminum, iron, magnesium and potassium, and also to compute the amount of each element present. A goodness-of-fit parameter is computed for each unknown sample, and it serves as an indication of the reliability of the final results.

Oxygen content was determined by a separate procedure which consists of five short irradiations and counts under standardized conditions. The observed activity is then compared to that of oxygen standards by a computer program, written to facilitate the quantitative calculations and to provide an error estimate.

This work was carried out with partial funding support under the NSF Sea Grant, Institutional Grant GH-26, made to Texas A\&M University. 
Major Element Composition of Selected Samples from Leg 5

\begin{tabular}{|c|c|c|c|c|c|c|c|c|c|}
\hline \multicolumn{4}{|c|}{ Sample Designation } & \multicolumn{6}{|c|}{ Composition, Wt \% } \\
\hline Hole & Core & Section & $\begin{array}{r}\text { Sampled } \\
\text { at }(\mathrm{cm})\end{array}$ & $\mathrm{O}$ & $\mathrm{Si}$ & $\mathrm{Fe}$ & $\mathrm{Al}$ & $\mathrm{Mg}$ & $\mathrm{K}$ \\
\hline 32 & 4 & 4 & 76 & $50.63 \pm 1.57$ & $29.38 \pm 0.29$ & $4.28 \pm 0.20$ & $7.22 \pm 0.16$ & $1.23 \pm 0.24$ & $6.47 \pm 1.12$ \\
\hline 32 & 10 & 2 & 120 & $45.67 \pm 2.51$ & $26.11 \pm 0.27$ & $6.33 \pm 0.22$ & $7.31 \pm 0.15$ & $3.42 \pm 0.26$ & $5.21 \pm 1.07$ \\
\hline 33 & 4 & 5 & 20 & $48.31 \pm 2.71$ & $28.02 \pm 0.30$ & $4.93 \pm 0.21$ & $7.74 \pm 0.16$ & $2.18 \pm 0.26$ & $4.77 \pm 1.14$ \\
\hline 33 & 10 & 5 & 100 & $49.14 \pm 1.49$ & $24.93 \pm 0.20$ & $4.34 \pm 0.14$ & $5.54 \pm 0.10$ & $1.83 \pm 0.17$ & $4.73 \pm 0.75$ \\
\hline 34 & 3 & 1 & 90 & $47.68 \pm 0.90$ & $17.10 \pm 0.16$ & $3.12 \pm 0.11$ & $4.87 \pm 0.09$ & $1.36 \pm 0.14$ & $4.91 \pm 0.62$ \\
\hline 34 & 10 & 5 & 20 & $49.57 \pm 5.06$ & $26.70 \pm 0.26$ & $3.85 \pm 0.17$ & $5.05 \pm 0.13$ & $1.80 \pm 0.21$ & $4.71 \pm 0.94$ \\
\hline 35 & 2 & 6 & $69-71$ & $42.93 \pm 2.65$ & $28.46 \pm 0.40$ & $5.44 \pm 0.29$ & $7.60 \pm 0.22$ & $2.73 \pm 0.36$ & N.D. \\
\hline 35 & 14 & 4 & 70 & $43.87 \pm 2.19$ & $27.82 \pm 0.50$ & $6.84 \pm 0.41$ & $9.83 \pm 0.29$ & $3.00 \pm 0.50$ & $2.44 \pm 1.99$ \\
\hline 36 & 1 & 5 & 52 & $44.75 \pm 1.98$ & $17.76 \pm 0.29$ & $4.40 \pm 0.24$ & $5.81 \pm 0.17$ & $1.74 \pm 0.29$ & $7.26 \pm 1.26$ \\
\hline 36 & 6 & 2 & 60 & $47.25 \pm 1.24$ & $13.68 \pm 0.18$ & $3.05 \pm 0.14$ & $4.04 \pm 0.10$ & $1.52 \pm 0.17$ & $5.39 \pm 0.75$ \\
\hline 36 & 12 & 6 & 95 & $45.38 \pm 0.51$ & $17.57 \pm 0.23$ & $4.74 \pm 0.19$ & $4.41 \pm 0.13$ & $2.04 \pm 0.22$ & $4.60 \pm 0.93$ \\
\hline 37 & 1 & 3 & 70 & $47.23 \pm 3.14$ & $24.32 \pm 0.57$ & $4.46 \pm 0.42$ & $7.91 \pm 0.32$ & $1.51 \pm 0.53$ & $7.85 \pm 2.36$ \\
\hline 37 & 4 & 5 & 40 & $45.80 \pm 1.71$ & $8.69 \pm 0.29$ & $27.85 \pm 0.60$ & $2.82 \pm 0.19$ & $1.02 \pm 0.40$ & $13.10 \pm 1.80$ \\
\hline 38 & 2 & 6 & 70 & $49.23 \pm 2.23$ & $23.45 \pm 0.32$ & $5.82 \pm 0.26$ & $7.91 \pm 0.19$ & $1.61 \pm 0.31$ & $8.50 \pm 1.37$ \\
\hline 38 & 6 & 4 & 80 & $46.78 \pm 3.68$ & $2.67 \pm 0.19$ & $13.86 \pm 0.48$ & $0.86 \pm 0.13$ & $0.76 \pm 0.30$ & $6.85 \pm 1.33$ \\
\hline 39 & 1 & 4 & 70 & $54.34 \pm 1.67$ & $23.03 \pm 0.35$ & $5.97 \pm 0.30$ & $7.80 \pm 0.20$ & $3.51 \pm 0.37$ & $9.22 \pm 1.53$ \\
\hline 40 & 1 & 3 & 20 & $49.77 \pm 2.38$ & $24.30 \pm 0.30$ & $8.54 \pm 0.27$ & $7.95 \pm 0.17$ & $1.25 \pm 0.29$ & $10.47 \pm 1.29$ \\
\hline 41 & 1 & 2 & 70 & $48.25 \pm 2.31$ & $24.90 \pm 0.24$ & $4.90 \pm 0.18$ & $7.95 \pm 0.14$ & $1.62 \pm 0.23$ & $8.50 \pm 1.01$ \\
\hline 42 & 10 & 2 & 70 & $54.04 \pm 3.33$ & $28.79 \pm 0.76$ & $2.08 \pm 0.38$ & $0.75 \pm 0.28$ & $0.78 \pm 0.40$ & $8.65 \pm 2.48$ \\
\hline
\end{tabular}

N.D. $=$ Not Detected 